\title{
TUMOR NEUROECTODÉRMICO MELANÓTICO DE LA INFANCIA. PRESENTACIÓN DE UN CASO CLÍNICO
}

\author{
MELANOTIC NEUROECTODERMAL TUMOR OF INFANCY. REPORT OF A CLINICAL CASE \\ TUMOR NEUROECTODÉRMICO MELANÓTICO DA INFÂNCIA. APRESENTAÇÃO DE UM CASO CLINICO
}

\section{MARÍA BETANCOURT RUIZ, ${ }^{1,2}$ VICKY RONQUILLO CAMPUZANO, ${ }^{2}$ MARÍA CEDEÑO JARAMILLO ${ }^{2}$}

\author{
1 Universidad Católica Santiago de Guayaquil, Guayaquil, Ecuador \\ ${ }^{2}$ Hospital de niños, Roberto Gilbert Elizalde, Guayaquil, Ecuador
}

\begin{abstract}
El tumor neuroectodérmico melanótico de la infancia es una rara condición que tiene su origen en la cresta neural, se han documentado alrededor de 486 casos desde que se lo describió por primera vez desde 1918, frecuentemente ubicado en el maxilar superior (60-80\%) y que se caracteriza por un rápido crecimiento. Se describe un caso femenino de un lactante menor de 5 meses de edad, que presentó tumefacción en región parietooccipital, de dos meses de evolución. La radiografía de cráneo mostró una imagen de neoformación ósea de bordes lobulados a nivel parietooccipital. La tomografía computarizada de cráneo evidenció una lesión hiperostósica parierto-occipital medial con áreas de osteólisis, sin realce con el medio de contraste; la resonancia magnética nuclear de cráneo reveló una tumoración isointensa en T1 y T2 supratentorial que desplaza el parénquima cerebral y el seno sagital. Se realizó una resección quirúrgica evidenciándose un tumor óseo, que infiltraba la duramadre, grisáceo, semiduro con trabéculas óseas, poco vascularizadas. El estudio anatomopatológico describió un tumor neuroectodérmico melanótico de la infancia.
\end{abstract}

PALABRAS CLAVE: tumor neuroectodérmico melánotico, cresta neural, neoplasia.

Abstract

Melanotic neuroectodermal tumor of infancy is a rare condition that originates in the neural crest. About 486 cases have been documented since it was first described in 1918. It is frequently located in the upper jaw (60-80\%) and it is characterized by rapid growth. This report is of a female case of an infant under 5 months, who presented two-month-evolution swelling in the parieto-occipital region. Skull radiograph showed an image of bone neoformation of lobulated edges at the parieto-occipital level. Tomography of the skull showed medial parierto-occipital hyperosotic injury with areas of osteolysis without contrast enhancement. Nuclear magnetic resonance of the skull revealed isointense tumor in T1 and supratentorial T2 that displaces the cerebral parenchyma and sagittal sinus. Surgical resection was performed, showing grayish, semi-hard bone tumor with undervascularized bone trabeculae that infiltrated the dura mater. Anatomopathological study described a melanotic neuroectodermal tumor of infancy.

KEYWORDS: neuroectodermal tumor, melanotic, neural crest, neoplasm.

Resumo

0 tumor neuroectodérmico melanótico da infância é uma rara condição que tem a origem na cresta neural. Há documentado cerca de 486 casos desde que foi descrito pela primeira vez desde 1918, frequentemente no maxilar superior (60-80\%) e que se caracteriza por um rápido crescimento. Se descreve um caso de uma paciente lactante de sexo feminino menor de 5 meses de idade, que apresentou tumefação em região parietooccipital, de dois meses de evolução. 0 raio x de crâneo mostrou uma imagem de neoformação óssea de fronteiras lobuladas a nível parieto-ocipital. A tomografia de crâneo revelou uma lesão hiperostosica parietooccipital medial com áreas de osteolisis, sem realce com o meio de contraste; a ressonância magnética nuclear de crâneo revelou um tumor isointenso em T1 e T2 supratentorial que desloca o parênquima cerebral e o seio sagital. Foi realizada uma resseção cirúrgica evidenciando um tumor ósseo infiltrando a dura-máter, grisaceo, semiduro com trabéculas ósseas, pouco vascularizadas. № estudo anatomopatológico descreveu um tumor neuroectodérmico melanótico da infância.

PALABRAS-CHAVE: tumor neuroectodermico, melanónito, crista neural, neoplasia. 
INTRODUCCIÓN

El tumor neuroectodérmico melanótico de la Infancia (TNMI), es benigno infrecuente, originado en la cresta neural y compuesto por células productoras de pigmentos (melanina). ${ }^{1}$ Fue descrito por primera vez en 1918 por Krompecher quien le dio el nombre de melanocarcinoma congénito; otros sinónimos incluyen: tumor neuroectodérmico pigmentado de la infancia, progonoma melanótico y odontoma epitelial melanótico. En 1966, Gorlin y Borello registraron niveles altos de ácido vanilmandélico (AVM), un marcador de tumores neurogénicos en estos pacientes por lo que se lo clasificó dentro del grupo de tumores de origen neurogénico. ${ }^{2}$ Se han documentado alrededor de 486 casos desde que se lo describió por primera vez hasta el año 2015. ${ }^{3}$ Se estima que cerca del $92.8 \%$ se presentan en la cabeza y el cuello, con mayor frecuencia en el maxilar superior (60-80\%), en el cráneo a nivel de la fontanela anterior más comúnmente $(10,8 \%)$, mandíbula $(6 \%)$ y en el cerebro $(4,3 \%){ }^{1}$

En raras ocasiones surgen en meninges, seno transverso, retina, mediastino, huesos, útero y epidídimo. Se presenta con mayor frecuencia (95\%) durante el primer año de vida, con una edad pico entre 2 y 6 meses de edad y se han reportado pocos casos en adultos. ${ }^{3}$

La literatura describe que no hay predilección de sexo, sin embargo una revisión de casos realizado por Kruse-Loser y colaboradores, en el año 2006, reportó un predominio en el sexo masculino. ${ }^{4}$ Clínicamente se presenta como una lesión de crecimiento rápido, no dolorosa, pudiendo provocar tumefacción de los tejidos blandos, compresión de las estructuras subyacentes y en caso de presentarse a nivel del maxilar superior, desplazamiento de los dientes. ${ }^{5}$

A continuación se describe el caso de una paciente con un TNMI a nivel de la región parietooccipital, de rara presentación por su ubicación anatómica.

\section{CASO CLÍNICO}

Lactante menor de 5 meses de edad de sexo femenino que acude al hospital Roberto Gilbert Elizalde el 20 de octubre de 2015, al área de consulta externa por presentar una masa protuberante indolora en la región parieto-occipital del cuero cabelludo de 2 meses de evolución, la cual se incrementó de tamaño progresivamente; durante el interrogatorio dirigido a la madre se niega antecedentes de trauma.

En la exploración física se palpa una masa firme, no dolorosa que mide aproximadamente de $3 \mathrm{~cm}$, sin cambios de coloración en la piel. No se observó déficit neurológico en la paciente. Es traslada al área de emergencia para su ingreso. Se realizó una radiografía simple de cráneo observándose imagen de neoformación ósea, de bordes lobulados a nivel parietooccipital. Los exámenes de laboratorio incluyeron hemograma completo, coagulación y bioquímica sanguínea encontrándose dentro de rangos normales.

La tomografía computarizada de cráneo (TC) evidenció una lesión hiperostósica pariertooccipital medial con áreas de osteolisis, sin realce con el medio de contraste. (Figura 1).

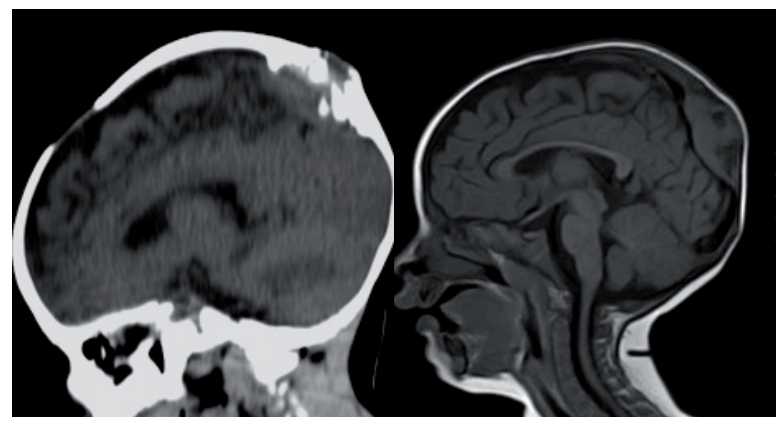

Figura 1. Tomografía simple de Figura 2. Resonancia de cerebro simple, cerebro. Corte sagital: se observa corte sagital en T1 se observa tumoración hiperostosis parieto-occipital de isointensa parieto-occipital que desplaza ubicación medial y áreas de osteolisis. el parénquima cerebral yel seno sagital. Fuente: departamento de imágenes del Fuente: departamento de imágenes del hospital Dr. Roberto Gilbert Elizalde. hospital Dr. Roberto Gilbert Elizalde.

La resonancia magnética nuclear (RMN) reveló una tumoración isointensa en T1 y T2 supratentorial que desplaza el parénquima cerebral y el seno sagital. (Figura 2). La paciente fue sometida a resección quirúrgica total de la lesión; durante el procedimiento quirúrgico se encontró una tumoración, que infiltraba la duramadre, grisácea, semidura, poco vascularizada.

La histopatología mostró trabéculas de células de cromatina clara y escaso citoplasma, en sectores se encontraron formaciones glanduliformes tapizadas por células de pequeño tamaño, otras áreas alveolares con marcado pigmento melánico. La proliferación involucró extensamente tejido conectivo fibroso denso. Estos datos morfológicos fueron compatibles con TNMI. (Figura 3 y 4). 


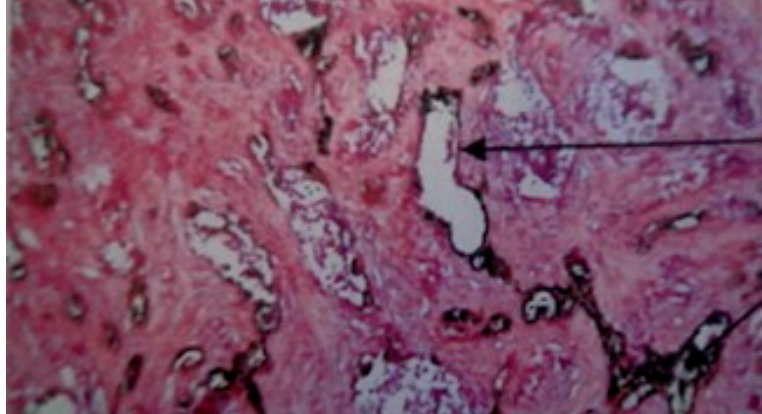

Figura 3. Anatomía patológica: Tumor neuroectodérmico melanótico de la infancia. Los cortes histológicos muestran una proliferación de estructuras tubulares irregulares tapizadas por células epiteliales poligonales intensamente cargadas de pigmento melánico.

Fuente: Departamento de Anatomía Patológica del hospital Dr. Roberto Gilbert Elizalde.

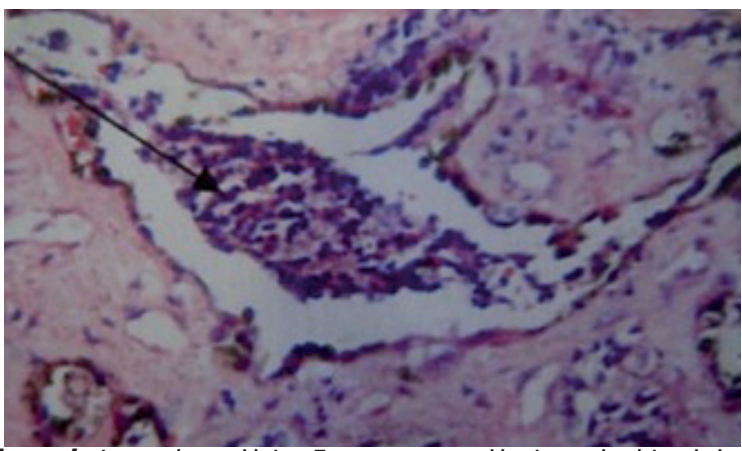

Figura 4. Anatomía patológica: Tumor neuroectodérmico melanótico de la infancia. Las estructuras proliferantes descritas se encuentran ocupadas en sus lúmenes por densos agregados de células pequeñas, redondas, de aspecto neuroblástico.

Fuente: Departamento de Anatomía Patológica del hospital Dr. Roberto Gilbert Elizalde.

La paciente permaneció hospitalizada durante 2 semanas posteriores a la cirugía, sin presentar complicaciones; egresó sin ningún déficit neurológico y estable clínicamente, siendo remitida a oncología para controles clínicos de forma bimensual, contando con un seguimiento imagenológico por medio de resonancia magnética a los 6 meses del egreso, (Figura 5) sin evidenciarse de recurrencia del tumor.

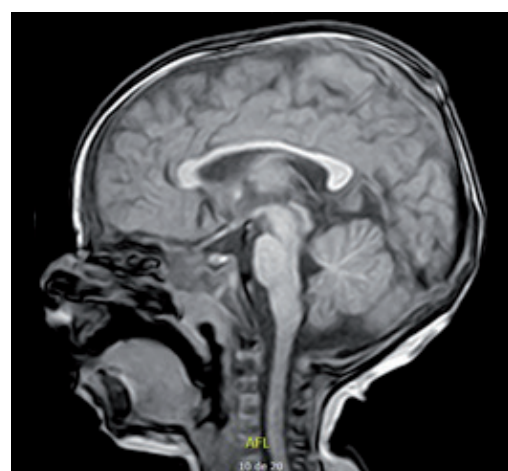

Figura 5. Resonancia de cerebro simple, corte sagital en T2: Ausencia de recidiva tumoral, 6 meses después del procedimiento quirúrgico. Fuente: Departamento de imágenes del hospital Dr. Roberto Gilbert Elizalde.
DISCUSIÓN

El TNMI es una neoplasia infrecuente que afecta a niños durante su primer año de vida, que es compatible con el presente caso ya que la paciente evidenció una protuberancia a los 5 meses de edad. Se la considera benigna; sin embargo 3-7\% de estos tumores tienen un comportamiento maligno. ${ }^{1-6}$

Macroscópicamente se observa como un tumor pigmentado que en algunos casos se podría confundir con una malformación vascular, microscópicamente se evidencia un estroma fibroso denso que contiene una población pequeña de células de neuroblastoma con núcleos hipercromáticos y citoplasma escaso; se encuentran células cúbicas de gran tamaño con contenido de melanina; lo cual coincidió con el informe emitido por el departamento de patología. ${ }^{5-7}$

En las radiografías convencionales se observa lesiones óseas radiolúcidas con o sin márgenes delimitados; si el tumor es grande se aprecia destrucción ósea y desplazamiento de estructuras adyacentes. La TC evidencia una lesión hiperdensa bien delimitada, aunque hay variantes que se describen como lesiones hipodensas. En la RMN la lesión suele ser hiperintensas en T1 e hipointensas en T2. ${ }^{7-10}$ Algunos pacientes tienen niveles altos AVM en orina y se asocia con tumores agresivos, sin embargo la sola presencia de este metabolito no es diagnóstica. ${ }^{5-11}$

La inmunohistoquímica evidencia positividad para enolasa neural específica, citoqueratina, HMB45 (human melanoma black 45), sinaptofisina, antígeno de membrana epitelial, proteína glial fibrilar acida y vimentina, pero es negativa a la proteína S-100. La expresión de Ki-67/CD99 es poco común en el TNMI, y podría asociarse con un crecimiento agresivo. ${ }^{5-12} \mathrm{El}$ diagnóstico diferencial del TNMI incluye: quistes (nasopalatino, glóbulo-maxilar); lesiones odontogénicas (odontomas, mixoma odontogénico); lesiones no odontogénicas no neoplásicas (granuloma central de células gigantes, malformación arteriovenosa, cefalohematoma); y lesiones neoplásicas no odontogénicas (rabdomiosarcoma, linfoma de Burkitt, sarcoma de Edwing, neuroblastoma), teniendo en cuenta que en los cuatro últimos no hay población celular polifenotípica con expresión neural, epitelial, ni con los marcadores melanocíticos característicos del TMNI, pudiendo descartarse teniendo en cuenta el cuadro clínico y las características radiológicas. ${ }^{5,12-14}$ 
La cirugía es el tratamiento de elección que ofrece una alta probabilidad de curación a largo plazo, ${ }^{15}$ y fue el tratamiento realizado con éxito en el paciente de este reporte. La quimioterapia es otra opción en caso de no realizarse la resección completa de la lesión o en caso de recidivas, que no fue requerida porque se realizó resección total del tumor. El TNMI presenta una tasa de recurrencia del 10 al 15\%.12-16 Rachidi y colaboradores, reportaron que la presentación en edades tempranas podría ser un indicador pronóstico de recurrencias. ${ }^{17}$

\section{CONCLUSIONES}

Aunque la mayoría de estos tumores son benignos, la tasa de recurrencias de $10-15 \%$ y la tasa de malignidad de $3-7 \%$ reportadas en la literatura, obligan a realizar un seguimiento oncológico. El diagnóstico precoz y el tratamiento quirúrgico mediante la resección total de la tumoración permiten el control con éxito a largo plazo del TNMI, evitando las recurrencias y repetidas cirugías con exéresis de la duramadre infiltrada inclusive. Por todo lo anterior es necesario el tratamiento en el momento adecuado para evitar complicaciones y metástasis.

\section{REFERENCIAS BIBLIOGRÁFICAS}

1. Cui Y, Mao ZHE, Liao C. Melanotic neuroectodermal tumor of infancy : A case report and review of the surgical treatment. Oncol Lett. 2014;9(1):29-34.

2. Mohanty SP, Ray JG, Mukherjee RS, Mandal C. Rare disease Melanotic neuroectodermal tumour of infancy. BMJ Case Rep. 2010;1-5.

3. Ma Y, Zheng J, Yang S, Zhu H, Dong K, Xiao X, et al. Case Report Melanotic neuroectodermal tumor of infancy in the soft tissue of the forearm : report of a case. Int J Clin Exp Pathol. 2015;8(10):13584-9.

4. Kruse-lösler B, Gaertner C, Bürger H. Melanotic neuroectodermal tumor of infancy: systematic review of the literature and presentation of a case. Oral Surg Oral Med Oral Pathol Oral Radiol Endod. 2006;102(2):204-16.

5. Nazir B, Gupta H, Chaturvedi AK, Rao SA, Jena A, Oncology PS, et al. Melanotic neuroectodermal tumor of infancy: discussion of a case and a review of the imaging findings. Cancer Imaging. 2009;9:121-5.
6. Albuquerque AF, Cunha JF, Avelar RL, Juca E, Costa FW, Macedo MS. Extensive Melanotic Neuroectodermal Tumor of Infancy. Head Neck Pathol. 2016;10(3):349-53.

7. Goswami M, Bhushan U, Mohanty S. Melanotic Neuroectodermal Tumor of Infancy. JClin Diagn Res. 2016;10(6):ZJ07-8.

8. 8. Haque S, McCarville MB, Sabire N, McHugh K. Melanotic neuroectodermal tumour of infancy: CT and MR findings. Pediatr Radiol. 2012;42(6):699-705.

9. 9. Jeon HJ, Kong DS, Shin HJ. Melanotic neuroectodermal tumor of infancy : a case report. Childs Nerv Syst. 2008;24(12):1489-92.

10. 10. Naidoo J, Potgieter L, Wieselthaler N, Pillay K. Melanotic neuroectodermal tumour of infancy, a rare cause for a bump on the head. Childs Nerv Syst. 2013;29(1):167-72.

11. 11. Reddy ER, Kumar MS, Aduri R, Sreelakshmi N. Melanotic neuroectodermal tumor of infancy: A rare case report. Contemp Clin Dent. 2013;4(4):55962.

12. 12. De Souza DF, Sendyk DI, Seo J, da Fonseca EV, Naclério-Homem Mda G, Deboni MC. Melanotic neuroectodermal tumor of infancy in the maxilla. Case Rep Dent. 2013;2013:726815.

13. 13. Khan $M B$, Soares $D$, Tahir MZ, Kumar R, Minhas K, Bari ME. Melanotic neuroectodermal tumour of infancy: a rare brain tumour of childhood. J Coll Physicians Surg Pak. 2013;23(5):367-9.

14. 14. Bangi BB, Tejasvi ML. Melanotic neuroectodermal tumor of infancy: A rare case report with differential diagnosis and review of the literature. Contemp Clin Dent. 2012;3(1):108-12.

15. 15. Lambropoulos V, Neofytou A, Sfougaris D, Mouravas V, Petropoulos A. Melanotic neuroectodermal tumor of infancy (MNT1) arising in the skull. Short review of two cases. Acta Neurochir (Wien). 2010;152(5):869-75.

16. 16. Agarwal P, Saxena S, Kumar S, Gupta R. Melanotic neuroectodermal tumor of infancy: Presentation of a case affecting the maxilla. J Oral Maxillofac Pathol. 2010;14(1):29-32.

17. Rachidi S, Sood AJ, Patel KG, et al. Melanotic Neuroectodermal Tumor of Infancy: A Systematic Review. J Oral Maxillofac Surg. 2015;73(10):1946-56. 